\title{
Bilateral peripheral facial palsy following asymptomatic COVID-19 infection: a case report
}

\author{
Jeroen Kerstens ${ }^{1}\left[\right.$ [ $\cdot$ Lien Deschuytere ${ }^{2} \cdot$ Katlijn Schotsmans $^{1} \cdot$ Emke Maréchal $^{1}$ (i)
}

Received: 13 January 2021 / Accepted: 26 March 2021 / Published online: 5 April 2021

(c) Belgian Neurological Society 2021

Keywords Bilateral facial palsy $\cdot$ Peripheral facial palsy $\cdot$ Facial diplegia $\cdot$ COVID-19 $\cdot$ SARS-CoV-2

\section{Dear Editor,}

The novel coronavirus disease-2019 (COVID-19), caused by severe acute respiratory syndrome coronavirus-2 (SARSCoV-2), has been associated with a broad spectrum of both central and peripheral neurological manifestations [1]. Neurological symptoms may be the presenting or even the only feature of the disease. Here, we present the first published case of isolated bilateral facial palsy (BFP) in the context of otherwise asymptomatic COVID-19.

A 27-year-old man in otherwise good health was scheduled for wisdom teeth extraction and according to local guidelines underwent a preoperative nasopharyngeal swab for SARS-CoV-2 PCR testing, which was positive. He had no respiratory or other symptoms at the time or in the days before testing and remained asymptomatic during the following days.

Five weeks later, he presented to the emergency department of another hospital with a one-day history of altered taste, reduced right eyelid closure and drooping of the right corner of the mouth. Right-sided peripheral facial palsy (House-Brackmann (HB) grade III) was diagnosed and the patient was discharged with a 10-day taper of oral methylprednisolone and artificial tears. Two days later, he returned to the same hospital because symptoms had worsened. On neurological examination, his facial palsy had progressed to grade IV without any additional abnormalities. A five-day course of valaciclovir was added to his treatment and he was again discharged. Three days later, he presented to the

Jeroen Kerstens

kerstensjeroen@gmail.com

1 Department of Neurology, Middelheim Hospital, Antwerp, Belgium

2 Department of Otorhinolaryngology, Middelheim Hospital, Antwerp, Belgium emergency department of our hospital because his symptoms had not improved and he had noticed reduced brow elevation and eyelid closure on the left side as well. Neurological examination showed asymmetrical bilateral peripheral facial palsy (HB grade $\mathrm{V}$ on the right and grade III on the left side) but was otherwise normal. The patient was admitted to the neurology department where the clinical picture progressed to bilateral grade $\mathrm{V}$ palsy over the following three days. Blood tests including complete blood count, renal and thyroid function, electrolytes, glucose, glycated hemoglobin, liver tests, serum electrophoresis, antineutrophil cytoplasmic, antiganglioside and antisulfatide antibodies, serology for human immunodeficiency virus, syphilis and Borrelia were all either normal or negative. Epstein-Barr virus (EBV) EBNA-IgG, herpes simplex virus (HSV) IgG and varicella zoster virus (VZV) IgG all were positive with negative IgM, consistent with previous infections. SARS-CoV-2 antibodies also turned out to be positive. Antinuclear antibodies were positive with a low titer (1:80) and without anti-double stranded DNA or other well-characterized antibodies. Serum angiontensin-converting enzyme (ACE) was slightly elevated (57 U/L, normal values $8.8-54.8$ ), possibly due to the patient's smoking habits. Chest X-ray was normal without hilar or mediastinal lymphadenopathy. Brain MRI on day 2 of hospitalization (7 days after symptom onset) showed bilateral contrast enhancement of the facial nerves without other abnormalities. Needle electromyography ( 8 days after symptom onset) showed no contraction in the right and markedly reduced contraction in the left facial muscles; nerve conduction studies of the right upper and lower limb ( 8 days after symptom onset) were normal without evidence for Guillain-Barre syndrome. Lumbar puncture (7 days after symptom onset) showed acellular cerebrospinal fluid with normal glucose and protein levels, a slightly elevated $\mathrm{IgG}$ (46 mg/L, normal values $10-30)$ and IgG-index (0.62, normal values $0.30-0.60$ ) and identical oligoclonal bands in 
serum and cerebrospinal fluid. Ear-nose-throat examination did not reveal any local infection, neoplasm or other cause for his symptoms. Since all other plausible causes had been ruled out, our final diagnosis was BFP following COVID-19. The patient finished his valaciclovir course and methylprednisolone taper and on follow-up, two months later, he had almost completely recovered to bilateral HB grade I.

BFP is rare, comprising $0.3-2 \%$ of all facial palsy cases [2]. Among the most important differential diagnoses are infectious (Lyme disease, infectious mononucleosis, HIV, syphilis), inflammatory (multiple sclerosis, sarcoidosis, Guillain-Barre syndrome) and metabolic causes (diabetes mellitus) [2], all of which were excluded in our patient. As the patient tested positive for HSV and VZV IgG, it cannot be ruled out that reactivation of one of these by COVID19 rather than COVID-19 itself was the underlying disease mechanism. The use of adjunctive antiviral therapy next to corticosteroids is controversial in idiopathic facial palsy but might be more readily considered when a clear viral cause is established, although even in these cases it is unclear if the nerve damage is due to a direct viral effect rather than an inflammatory response. While both unilateral and BFP associated with COVID-19 have been previously reported [3-5], all BFP cases so far also had additional symptoms and/or facial weakness in the setting of Guillain-Barre syndrome $[4,5]$.

To our knowledge, this is the first published case of isolated BFP in the context of otherwise asymptomatic COVID19. Notably, this case meets the provisional case definition of 'probable association' of COVID-19 with neurological disease as established by Ellul et al. in their recent review [1]. We recommend that in a patient with BFP, COVID-19 should be ruled out even if the patient lacks other COVID19-related symptoms, in order to take the adequate isolation measures to prevent further transmission of this contagious disease. In patients with a known recent SARS-CoV-2 infection presenting with BFP, it should be considered in the differential diagnosis, but other causes should be ruled out.

Author contributions JK drafted the manuscript and took care of the patient; LD, KS and EM took care of the patient and critically reviewed the manuscript.
Funding None.

\section{Declarations}

Conflict of interest The authors declare that they have no conflict of interest.

Ethical approval Ethics approval was waived as all procedures that were performed were part of routine clinical care.

Informed consent The patient provided written informed consent for the publication of his anonymized data.

Consent for publication Written informed consent of the patient was obtained.

Availability of data and material Data available on request from the authors.

\section{References}

1. Ellul MA, Benjamin L, Singh B, Lant S, Michael BD, Easton A, Kneen R, Defres S, Sejvar J, Solomon T (2020) Neurological associations of COVID-19. Lancet Neurol 19:767-783. https:// doi.org/10.1016/S1474-4422(20)30221-0

2. Jain V, Deshmukh A, Gollomp S (2006) Bilateral facial paralysis: case presentation and discussion of differential diagnosis. J Gen Intern Med 21(7):C7-C10. https://doi.org/10.1111/j.1525-1497. 2006.00466.x

3. Lima MA, Silva MTT, Soares CN, Coutinho R, Oliveira HS, Afonso L, Espíndola O, Leite AC, Araujo A (2020) Peripheral facial nerve palsy associated with COVID-19. J Neurovirol 26:941-944. https://doi.org/10.1007/s13365-020-00912-6

4. Paybast S, Gorji R, Mavandadi S (2020) Guillain-Barré Syndrome as a neurological complication of Novel COVID-19 infection: a case report and review of the literature. Neurologist 25(4):101103. https://doi.org/10.1097/NRL.0000000000000291

5. Cabrera Muras A, Carmona-Abellán M, Collía Fernández A, Uterga Valiente JM, Antón Méndez L, García-Moncó JC (2020) Bilateral facial nerve palsy associated with COVID-19 and Epstein-Barr virus co-infection. Eur J Neurol. https://doi.org/10. 1111/ene.14561

Publisher's Note Springer Nature remains neutral with regard to jurisdictional claims in published maps and institutional affiliations. 